\title{
Marker-assisted introgression of 4 Phytophthora capsici resistance QTL alleles into a bell pepper line: validation of additive and epistatic effects
}

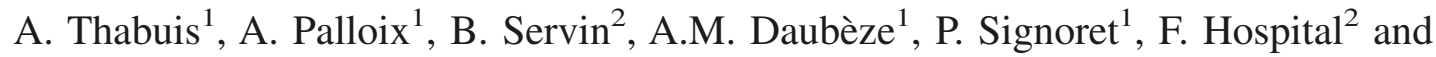 \\ V. Lefebvre ${ }^{1, *}$ \\ ${ }^{1}$ INRA, Genetics and Breeding of Fruits and Vegetables, BP94, 84143 Montfavet cedex, France; ${ }^{2}$ INRA, UMR \\ de Génétique Végétale, Ferme du Moulon, 91190 Gif sur Yvette, France; *Author for correspondence (e-mail: \\ veronique.lefebvre@avignon.inra.fr; tel: +33 (0)4 327228 06; fax: +33 (0)4 327227 02)
}

Received 3 June 2003, accepted in revised form 13 November 2003

Key words: Capsicum annuum L., Disease resistance, Epistasis, Horticultural traits, Marker-assisted selection, QTL

\begin{abstract}
The aim of the present study was to transfer resistance to $P$. capsici alleles at four quantitative trait loci (QTLs) from a small fruited pepper into a bell pepper recipient line using markers. The marker-assisted selection program was initiated from a doubled-haploid line issued from the mapping population and involved three cycles of marker-assisted backcross (MAB). Two populations, derived by selfing the plants selected after the first selection cycle, were genotyped and evaluated phenotypically for their resistance level. The additive and epistatic effects of the four resistance factors were re-detected and validated in these populations, indicating that introgression of 4 QTLs in this MAB program was successful. A decrease of the effect for the moderate-effect QTLs and of the epistatic interaction was observed. Phenotypic evaluations of horticultural traits were performed on sample of each backcross generation. The results indicated an efficient return to the recipient phenotype using this MAB strategy.
\end{abstract}

\section{Introduction}

Phytophthora capsici, causing root rot and shoot blight, is one of the most devastating field or greenhouse diseases of pepper worldwide. This soilborne Oomycete is able to attack the plant at any developmental stage causing sudden wilt and the collapse of the plant. Soil chemical treatments have technical limitations and would be progressively banned because of environmental legislations. Breeding for $P$. capsici resistance remains a challenge. Several sources of resistance were described in intraspecific pepper germplasm but all displayed a partial effect and were found in exotic accessions. Among them, Perennial is an Indian line displaying a polygenic re- sistance (Lefebvre and Palloix 1996) but is smallfruited and pungent.

The polygenic resistance to $P$. capsici was dissected into 4 resistance components using 2 phenotypic tests performed in controlled conditions. Three resistance components (REC: receptivity, IND: inducibility and STA: stability) were quantitatively evaluated thanks to the stem inoculation procedure and revealed different steps of the adult plant-pathogen interaction (Pochard et al. 1976; Pochard and Daubèze 1980). The Root Rot Index component (RRI) was a semi-quantitative criterion based on the evaluation of the resistance after root inoculation of young plantlets (Palloix et al. 1988).

Many pepper breeding programs focused on breeding for resistance to $P$. capsici into large fruited cul- 
tivars. However, they were not fully successful since the released varieties displayed only a weak resistance level. In order to enhance the global resistance level, Palloix et al. (1990) initiated a first phenotypic recurrent breeding scheme to accumulate resistance factors from distinct accessions. A second phenotypic recurrent breeding scheme was set up to transfer the polygenic resistance into a favourable genetic background (Palloix et al. 1997). Three to six selection cycles enabled to transfer an intermediate resistance level into a large fruited pepper. However, attempts to transfer a higher resistance level decreased the genetic advance for horticultural traits.

The advent of molecular markers enabled to identify the chromosomal regions involved in the variation of the components of the $P$. capsici resistance of Perennial and to estimate their individual effects. This analysis was conducted using 114 doubled haploid $(\mathrm{DH})$ lines issued from the cross Perennial $\times$ Yolo Wonder (YW). A total of 5 different genomic regions displayed an additive effect on resistance. Four major epistatic relationships were detected between either additive QTLs or between QTLs involved only in epistatic relationships (Lefebvre and Palloix 1996; Thabuis et al. 2003).

Marker-assisted selection appeared as a promising tool for breeding quantitative resistance. Regarding the genetic distance between Perennial and bell pepper accessions (Lefebvre et al. 2001), the marker-assisted backcross strategy (MAB) appeared as the most suitable to transfer a limited number of QTLs. However, given the imprecision around the positions of the QTLs, Hospital and Charcosset (1997) showed that MAB needed to be optimised for a successful QTL transfer. They proposed a two-fold strategy: (i) selection for the donor alleles on the carrier chromosomes (foreground selection) and (ii) in the remaining plants, selection for the return to the recipient parent (background selection). Through their theoretical study, they showed that three markers spread along the confidence interval of each QTL enabled an efficient control of the QTLs during the introgression. Once the interval lengths and marker locations were defined, they computed the minimal population size for recovering at least one plant having the entire donor segments for a given type I error.

We conducted a MAB program to transfer favourable alleles at the 4 main QTLs controlling resistance to $P$. capsici from Perennial accession into $\mathrm{YW}$, a bell pepper line, by taking into account the optimisations from Hospital and Charcosset (1997). To speed up the

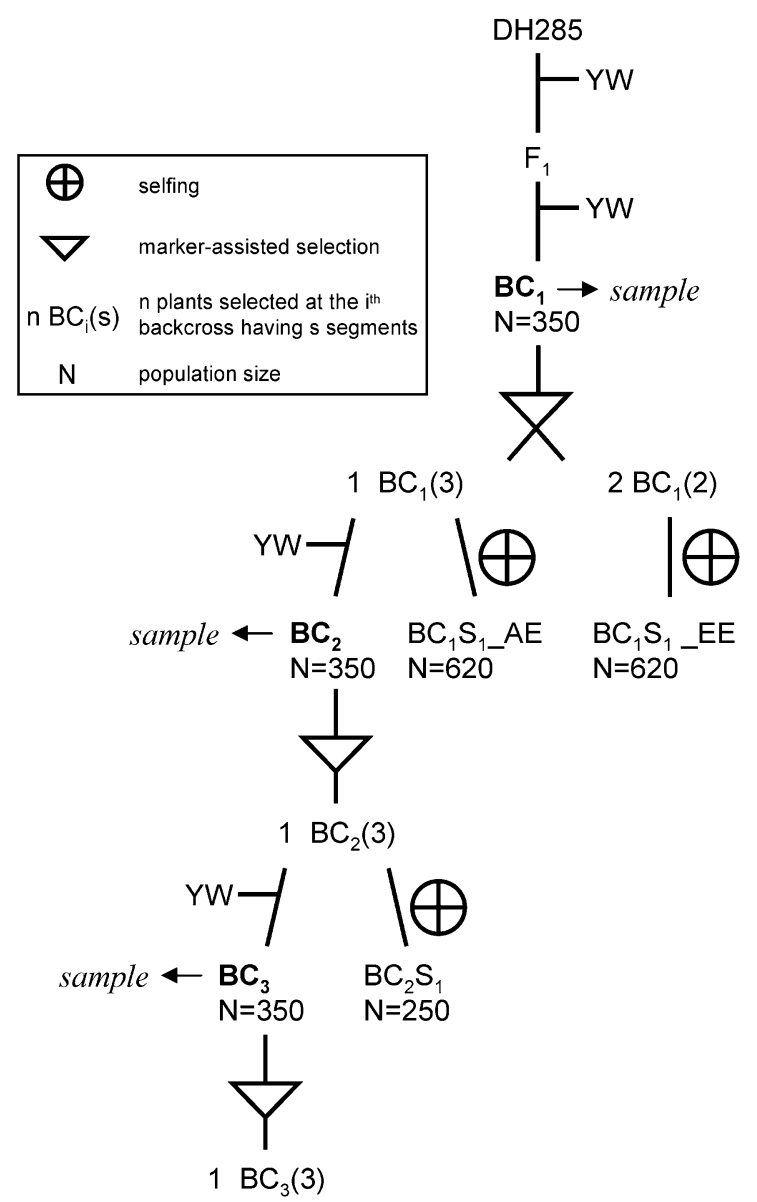

Figure 1. Synoptic view of the experimental study performed.

breeding process, a DH line from the mapping population, having all the chromosomal regions to be transferred (Thabuis et al. 2003), was used as the donor parent to initiate the MAB program.

In this paper, we examined (i) the results of $3 \mathrm{MAB}$ cycles conducted according to the theoretical optimisations, (ii) the additive and epistatic effects of the transferred segments in validation populations, and (iii) the impact of the background selection step on the improvement of horticultural traits.

\section{Materials and methods}

Plant material and breeding scheme

Backcross populations

DH285, a DH line issued from the initial mapping population, was chosen as the donor parent (Figure 
$1)$ : it possessed the 4 favourable alleles to be transferred and a YW genome content of $3.6 \%$ on the QTL-carrier chromosomes and $43.8 \%$ on the noncarrier chromosomes (Lefebvre et al. 2002). Two of the favourable alleles to be transferred were linked (approximately $20 \mathrm{cM}$ ) on chromosome P5 so that they were considered as a single segment during the MAB process whereas the 2 others mapped on $\mathrm{P} 2$ and P10. To increase the success to transfer favourable alleles at the QTLs, the 3 QTL-carrier chromosomes of the DH285 line are almost entirely of the Perennial phase (Figure 2). As indicated in Figure 1, three MAB cycles were performed. The $\mathrm{BC}_{1}, \mathrm{BC}_{2}$ and $\mathrm{BC}_{3}$ populations were firstly screened with markers linked to and in coupling with the $4 P$. capsici resistance alleles at the QTLs, and finally for the recovery of the recipient genetic background (as described below in molecular analyses).

\section{QTL validation populations}

Two validation populations were derived from the backcross populations in order to evaluate accurately the QTL effects in the recipient genetic background (Figure 1). A large progeny, named $\mathrm{BC}_{1} \mathrm{~S}_{1}$ AE, composed of 620 plants, was derived by selfing the plant selected after the first MAB cycle, containing the 4 resistance alleles at the QTLs. This population was genotyped with the QTL markers and was evaluated phenotypically for $P$. capsici resistance level, in order to evaluate QTL additive effects. A second validation population was aimed at evaluating the epistatic relationship between the 2 additive QTLs on P5 and P10. Two $\mathrm{BC}_{1}$ plants $\left(\mathrm{BC}_{1} 251\right.$ and $\mathrm{BC}_{1}$ 301) carrying both segments on P5 and P10 involved in interaction and the most efficient return to the recipient parent were used. A progeny of 620 plants, named $\mathrm{BC}_{1} \mathrm{~S}_{1}$ EEE, was derived by selfing these two $\mathrm{BC}_{1}$ plants. It was genotyped with markers of the P5 and P10 QTLs, and assessed for $P$. capsici resistance level. In addition, the resistance level of the selfed progeny of the selected $\mathrm{BC}_{2}$ plants $\left(\mathrm{BC}_{2} \mathrm{~S}_{1}\right)$ was assessed to evaluate the performance of the MAB strategy.

\section{Resistance evaluation}

The moderately aggressive $P$. capsici strain S101 was used for phenotypic assays (Lefebvre and Palloix 1996). It was maintained as described by Clerjeau et al. (1976). P. capsici resistance was evaluated using the stem inoculation test. The plants were decapitated at the $6^{\text {th }} / 7^{\text {th }}$-leaf stage and a mycelium plug was placed on the fresh section of the stem. The plants were kept in growth chambers under the conditions described in Pochard and Daubèze (1980). The measure of length necrosis during 21 days supplied 3 resistance components, as described by Lefebvre and Palloix (1996). Receptivity (REC, mm.d ${ }^{-1}$ ) measured the pathogen spread in early infection process $\left(3^{\text {rd }}\right.$ day post inoculation, DPI). Inducibility (IND, mm.d $\mathrm{d}^{-2}$ ) measured the deceleration of the necrosis length between the $3^{\text {rd }}$ and the $10^{\text {th }}$ DPI. Stability (STA, $\mathrm{mm} \cdot \mathrm{d}^{-1}$ ) measured the average speed of necrosis length between the $14^{\text {th }}$ and the $21^{\text {st }}$ DPI. The lower the value of the resistance component, the higher the resistance level. In all tests, controls were YW, Perennial, DH285 and PI201234 (10 plants per genotype).

\section{Horticultural trait evaluation}

The horticultural traits of samples from each backcross generation $(\mathrm{BC})$ were evaluated. The $\mathrm{BC}_{1}$ and $\mathrm{BC}_{2}$ populations were evaluated in 2000 and 2001. The $\mathrm{BC}_{3}$ population was evaluated only in 2001 . Every year, trials were placed in 2 cultivation conditions: under cold plastic greenhouse conditions from September to January at Almeria (Spain), and under field conditions from July to November in Sicily (Italy). For each BC population, 50 sampled plants were evaluated in a complete randomised design of 5 blocks of 10 plants. For the control inbred lines, 5 blocks of 5 plants each were included. The controls were YW, Perennial, and DH285.

The horticultural traits evaluated were the length of the main axis ( $\mathrm{AL}$ in $\mathrm{cm}$, from cotyledon to the $1^{\text {st }}$ flower) and the number of leaves (NL) on this axis which permitted the calculation of the internode length $(\mathrm{IL}=\mathrm{AL} / \mathrm{NL})$. For the fruit traits, 5 to $10 \mathrm{ma}-$ ture fruits were harvested from each plant and weighted together. The average fruit weight per plant (AFW in $\mathrm{g}$ ) was computed. The most representative fruit from each plant was chosen to measure the fruit length (FL in $\mathrm{mm}$ ) and fruit width (FW in $\mathrm{mm}$ ), the fruit flesh thickness (FT in $\mathrm{mm}$ ) and to calculate the fruit shape $(\mathrm{FS}=\mathrm{FL} / \mathrm{FW})$. All these horticultural traits clearly discriminated Perennial from YW.

\section{Molecular data}

The DNA was extracted using the microprep protocol described by Fulton et al. (1995). For the foreground 

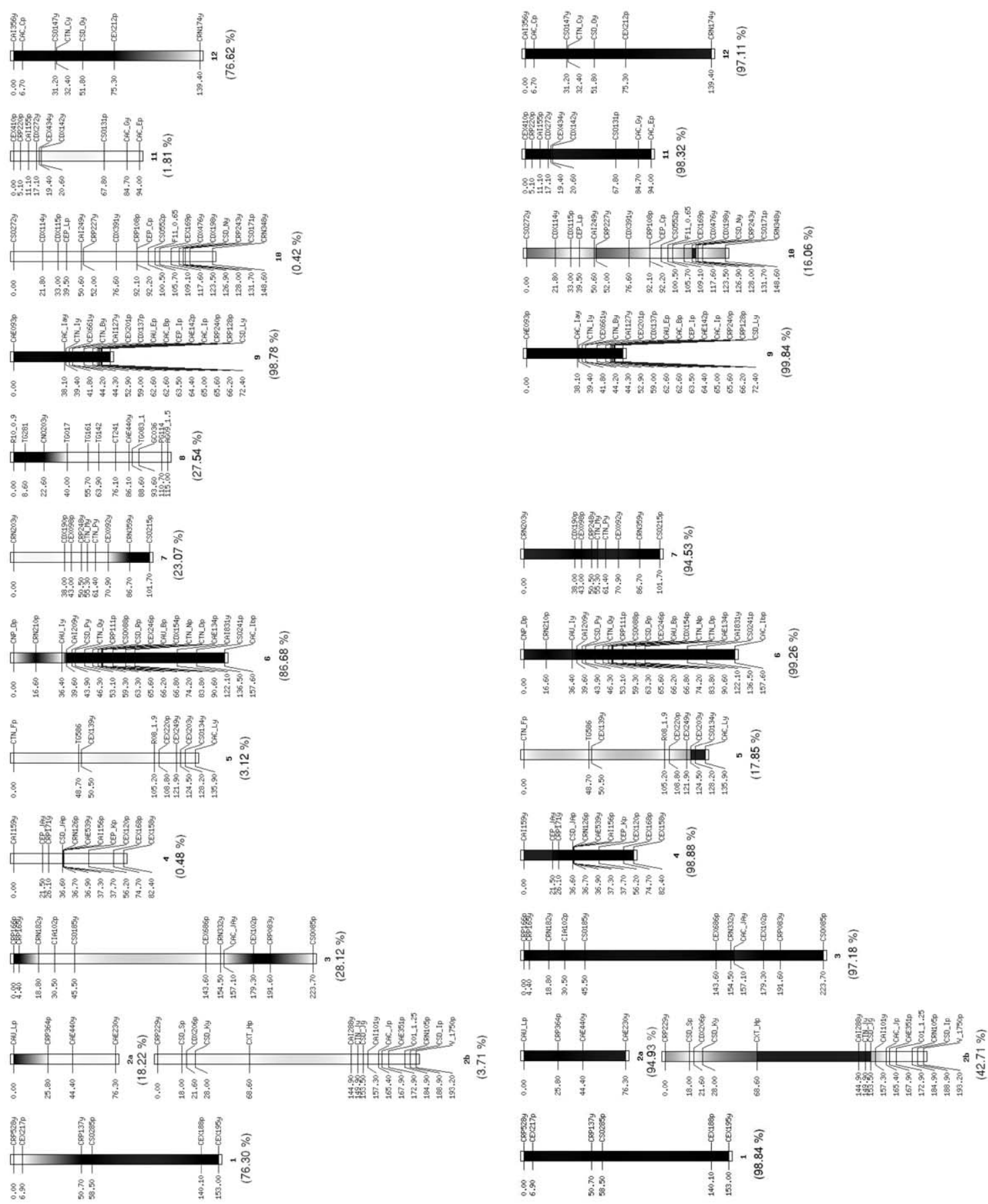

Figure 2. Precision graphical genotypes of the double haploid line (DH285, top) used as the donor parent and the selected $\mathrm{BC}_{3}$ individual (bottom). Note: Chromosomes are shaded according to the genotype of the individuals. The grey scales reflect the probability that the individual is homozygous Yolo Wonder, from black (probability of 1) to white (null probability). Proportion of YW genome is indicated between parentheses under each chromosome.

selection step, 3 to 4 markers (3 for QTLs on P5 and P10, 4 for the QTL on P2) were used for controlling each segment. One marker was located close to the most likely position of the QTL while the others were 

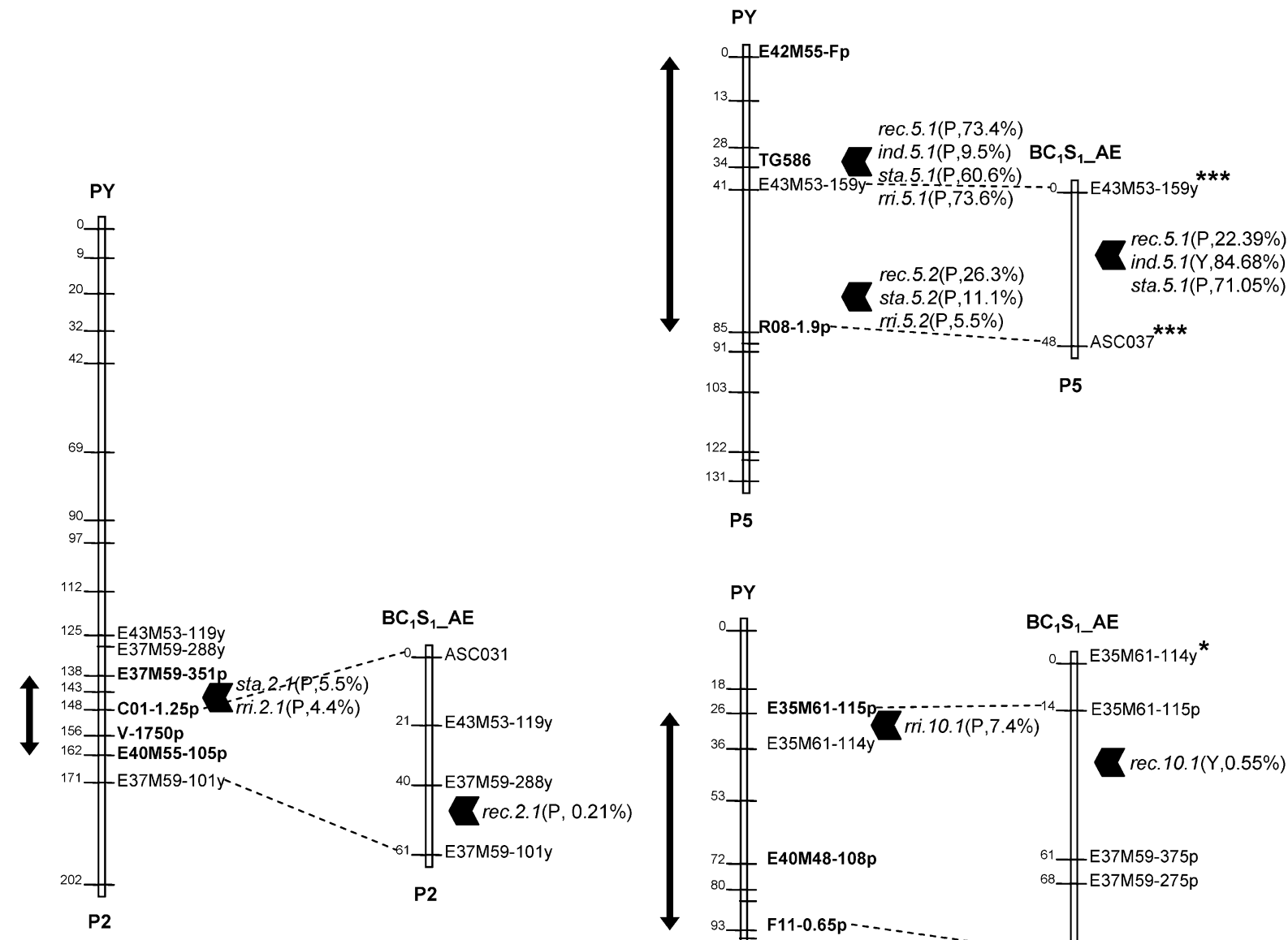

P5

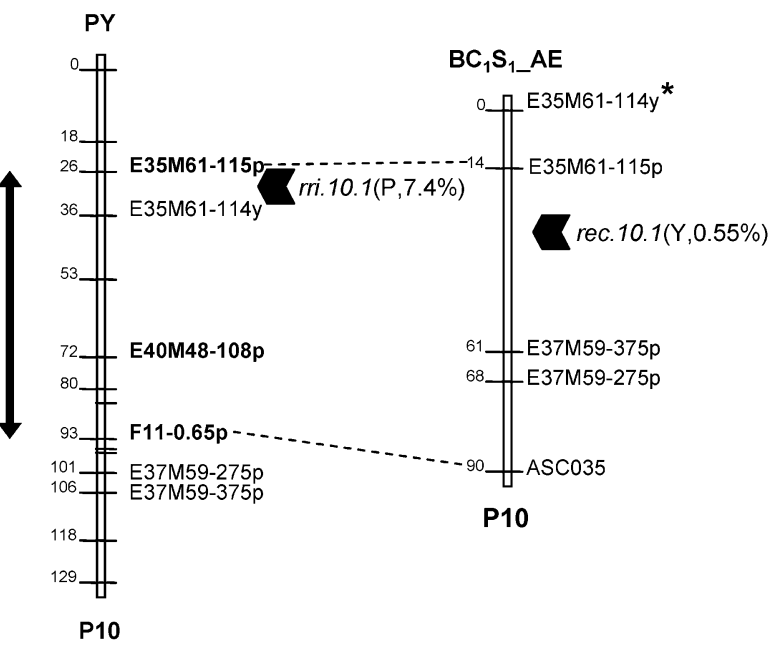

Figure 3. Location on the map 'PY' of the 3 chromosomal segments transferred through the MAB program and effects detected in the $\mathrm{BC}_{1} \mathrm{~S}_{1} \_\mathrm{AE}$ validation population. Note: The markers indicated in bold were used for controlling the transferred segments. The vertical black arrows indicate the segments transferred. The stars indicate the markers displaying a significant effect on a resistance component through one-way ANOVA (for details, see Table 2). The significance values are *: $0.01<\mathrm{P}<0.05, * *: 0.0001<\mathrm{P}<0.01, * * *$ : $\mathrm{P}<0.0001$. The horizontal black arrows represent the most likely QTL positions (Interval Mapping results for the $\mathrm{BC}_{1} \mathrm{~S}_{1} \_\mathrm{AE}$ population, Composite Interval Mapping results for the PY population). "rec" corresponds to the receptivity, "ind" to the inducibility, "sta" to the stability, and "rri" to the root rot index as defined by Thabuis et al. (2003). PY represents the doubled haploid line mapping population and $\mathrm{BC}_{1} \mathrm{~S}_{1} \mathrm{AE}$ the validation population. The markers are named according to Lefebvre et al. (2002) and the QTLs according to Thabuis et al. (2003). Distances are expressed in centimorgan (Haldane).

controlling the QTL position support interval defined by a LOD drop-off of 1.5 (Figure 3). A total of 10 markers were used for the selection of the 4 resistance QTLs. Markers included 3 RAPDs (further converted into 1 SCAR and 2 CAPSs), 5 AFLPs, 1 ISSR and 1 RFLP. The dominant markers used were in coupling with the resistant alleles at the QTLs. The RAPD markers R08_1.9p, C01_1.25p and F11_0.65p, re- vealed as described by Lefebvre et al. (1995), were used for the first MAB cycle. For the two other MAB cycles, R08_1.9p and C01_1.25p were converted into codominant CAPS markers (ASC037 and ASC031, respectively) whereas F11_0.65p was converted into a dominant SCAR marker (ASC035p). Five AFLP markers, obtained as described by Vos et al. (1995) and named E35M61-115p, E37M59-351p, E40M48- 
108p, E40M55-105p and E42M55-Fp, were used to assess the presence of the favourable alleles at the QTLs. The RFLP marker TG586 was revealed as described by Lefebvre et al. (1993) using EcoRI as a restriction enzyme. The other markers provided by the 5 previous AFLP primer combinations and 11 additional ones (E37M54, E37M60, E37M62, E38M48, E38M61, E39M48, E39M49, E41M49, E41M51, E43M53 and E45M58) were used for the background selection.

For the $\mathrm{BC}_{1} \mathrm{~S}_{1}$ AE population, the 3 specific PCR markers as well as 8 AFLP markers generated by 3 primer combinations (E37M59, E43M53 and E35M61) were assayed. These 8 markers mapped to the confidence interval of the QTLs transferred and were presented on Figure 3.

For the $\mathrm{BC}_{1} \mathrm{~S}_{1}$ EE population, 2 specific PCR markers, that mapped to both regions displaying interactions (ASC037 on P5 and ASC035p on P10), and the AFLP marker E40M55-104y, that mapped to P10, were assayed.

\section{Statistical analyses}

\section{Plant selection}

The molecular data from the MAB program were used for selecting plants carrying the donor alleles at the 3 introgressed intervals and displaying the best return to the recipient parent. To estimate more accurately the return to the recipient parent, the precision graphical genotypes were performed using 'MDM' software elaborated by Servin et al. (2002) which computes the probability of the donor allele presence in each point of the genome. 'GRAFGEN' software was used for 'precision graphical genotypes' design, according to the results of MDM (Servin and Hospital, Submitted, http://moulon.inra.fr/ servin/grafgen).

\section{Validation of the additive and epistatic QTL effects} The additive QTL validation was performed in $\mathrm{BC}_{1} \mathrm{~S}_{1}$ AE by one-way ANOVA where the phenotypic resistance value is explained by a single-marker effect, with PROC GLM of the SAS package (SAS Institute, 1989). The 11 markers assayed for the validation step were mapped with a LOD threshold of 3 and a maximum recombination rate of 0.3 using 'Mapmaker' software (Lander et al. 1987). The QTL validation was also performed using Interval Mapping (IM) method with 'QTL Cartographer' software (Basten et al. 1997), that provided the most likely position of the QTLs, their effects $\left(\mathrm{R}^{2}\right)$ and the additive and dominance effects $(\mathrm{a}, \mathrm{d})$. The dominance ratio Id/al was also estimated for each QTL. The phenotypic means for the 3 resistance components were computed for each genotypic class using PROC GLM and 'lsmeans' option, and compared using 'tdiff' option with a type I error of $5 \%$.

The digenic interaction effect between ASC037 on P5 and 2 markers on P10 (ASC035p and E40M55$104 y)$ was tested using a 2-way ANOVA in the $\mathrm{BC}_{1} \mathrm{~S}_{1}$ EE population with two additive marker effects and an interaction factor between both markers (PROC GLM). As the 2 markers used for P10 checking were dominant and originated from distinct allelic phases, it enabled to deduce the genotypic class at the P10 QTL (in the absence of recombination events). The phenotypic means for the 3 resistance components were computed for each genotypic class ('lsmeans' option) in order to compare the 9 allelic combinations ('tdiff' option with a type I error of $5 \%$ ).

\section{Phenotypic analysis of the BC populations}

Resistance evaluation of the $\mathrm{BC}_{1} \mathrm{~S}_{1} \_\mathrm{AE}$ and $\mathrm{BC}_{2} \mathrm{~S}_{1}$ populations were performed at 2 different years. Thus the individual plant values were adjusted to the control values (PROC REG) after checking the variance homogeneity. A one-way ANOVA was performed where the resistance component value of a plant is explained by the single MAB cycle effect (PROC GLM). The adjusted means per cycle were computed and compared for the 3 resistance components using 'lsmeans' and 'tdiff' options with a type I error of 5\%.

The main source of variation affecting horticultural traits was analysed on the data collected from the 4 trials (2 years, 2 locations) with the following ANOVA model: Pijkl $=\mu+\mathrm{Bi}+\mathrm{Lj}+\mathrm{Yk}+(\mathrm{BX}-$ $\mathrm{L}) \mathrm{ij}+(\mathrm{BXY}) \mathrm{ik}+\mathrm{Rijkl}(\mathrm{PROC}$ GLM), where Pijkl is the horticultural trait value of the plant $1, \mathrm{Bi}$ the effect of the backcross population $\mathrm{i}, \mathrm{Lj}$ the effect of the location j, Yk the effect of the year k, (BXL)ij the interaction factor effect between the backcross population $\mathrm{i}$ and the location $\mathrm{j},(\mathrm{BXY}) \mathrm{ik}$ the interaction factor effect between the backcross population $\mathrm{i}$ and the year k, Rijkl the residual effect. Trials were also analysed separately, for each location and each year with the following ANOVA model: Pijk $=\mu+\mathrm{Bi}+$ $\mathrm{bj}+(\mathrm{BXb}) \mathrm{ij}+\mathrm{Rijk}$ (PROC GLM), where bj is the effect of the block $\mathrm{j}$, and $(\mathrm{BXb}) \mathrm{ij}$ is the interaction factor effect between the backcross population $\mathrm{i}$ and the block j. An effect was declared significant if $\mathrm{P}<0.05$. For each backcross generation sample, as 
Table 1. Number of plants selected at each step of the sequential approach of the 3 MAB cycles

\begin{tabular}{|c|c|c|c|c|c|c|}
\hline & \multirow{4}{*}{$\begin{array}{l}\text { Initial number of } \\
\text { plants }\end{array}$} & \multicolumn{4}{|c|}{ Foreground selection on QTL-carrier chromosomes } & \multirow{3}{*}{$\begin{array}{l}\text { Background se- } \\
\text { lection } \\
\text { Step } 3 \\
\text { on non QTL-car- } \\
\text { rier chromosomes }\end{array}$} \\
\hline & & \multicolumn{3}{|l|}{ Step 1} & \multirow{2}{*}{$\begin{array}{l}\text { Step } 2 \\
\mathrm{P} 5+\mathrm{P} 10+\mathrm{P} 2 \text { (all } \\
\text { the } \mathrm{CI}^{2} \text { ) }\end{array}$} & \\
\hline & & P5 & P10 & $\mathrm{P} 2$ & & \\
\hline & & $\begin{array}{l}\text { R08_1.9p } \\
(\text { ASC037) }\end{array}$ & $\begin{array}{l}\text { F11_0.65p } \\
\text { (ASC035p) }\end{array}$ & $\begin{array}{l}\text { C01_1.25p } \\
(\mathrm{ASC} 031)\end{array}$ & $\begin{array}{l}5 \text { AFLP }+1 \\
\text { ISSR }+1 \text { RFLP }\end{array}$ & 2 to $11 \mathrm{PC}^{1}$ \\
\hline $\mathrm{BC}_{1}$ & 350 & 173 & 73 & 34 & 5 & 1 (2 PC) \\
\hline $\mathrm{BC}_{2}$ & 350 & 148 & 71 & 29 & 6 & 1 (11 PC) \\
\hline $\mathrm{BC}_{3}$ & 350 & 164 & 78 & 36 & 8 & 1 (11 PC) \\
\hline
\end{tabular}

${ }^{1} \mathrm{PC}$ : AFLP primer combinations, ${ }^{2} \mathrm{CI}$ : confidence interval

for the controls, the means were computed ('lsmeans' option) and compared ('tdiff' option with a type I error of $5 \%$ ).

\section{Results}

\section{Optimisation of the MAB strategy}

The markers used during the MAB program were those that maximised the probability of recovering the donor favourable alleles at the 3 target regions based on the computations of Hospital and Charcosset (1997). For the 3 transferred segments, this probability was assessed to $91 \%$ for the $1^{\text {st }}$ MAB cycle; for the 3 MAB cycles, this probability is above $88 \%$. The minimal population size based on the computations of Hospital and Charcosset (1997) was of 280 plants for a type I error of $1 \%$. The population size was increased to 350 plants during the 3 MAB cycles to enhance the probability of transferring the favourable alleles at the QTLs during the first selection cycle while maintaining a high probability for the next 2 selection cycles. This large population size also enabled an increase in the background selection rate (see below).

In order to minimise the genotyping effort, a sequential approach in 3 steps was adopted (Table 1). In a first step, only one marker per QTL was checked. The PCR marker linked to the resistant segment on chromosome P5 was firstly assayed. Only the plants carrying the Perennial allele (resistant) at this marker were further evaluated with the PCR marker of the P10-QTL. Then, the P5+P10-resistant allele plants were screened with the PCR marker of the P2-QTL. In a second step, the AFLP and RFLP markers were assayed on the reduced-size progeny in order to check for the integrity of the transferred segments. This strategy decreased the genotyping effort from 3500 data points ( 10 markers $\times 350$ plants $)$ to $772-844$ data points depending on the selection cycle. In a third step, the remaining plants were screened for their return to the recipient parent genome using AFLP markers regularly spread on the genome. After the third step, a single plant was selected and backcrossed to $\mathrm{YW}$.

\section{Effect of the 3 transferred segments}

Evaluation of the 3 components of resistance to $P$. capsici showed that the means of the $\mathrm{BC}_{1} \mathrm{~S}_{1} \mathrm{AE}$ and $\mathrm{BC}_{2} \mathrm{~S}_{1}$ populations were significantly lower (more resistant) than $\mathrm{YW}$ as a result of the transfer of the resistance factors. The $\mathrm{BC}_{1} \mathrm{~S}_{1} \mathrm{AE}$ population means were not significantly different from $\mathrm{DH} 285$ means while the $\mathrm{BC}_{2} \mathrm{~S}_{1}$ population was significantly less resistant than $\mathrm{DH} 285$. For the standardized resistance values, the $\mathrm{BC}_{2} \mathrm{~S}_{1}$ population was less resistant than the $\mathrm{BC}_{1} \mathrm{~S}_{1}$ AE population for IND and STA, while the means were not significantly different at $1 \%$ for REC. However, comparing the top 5\% in both validation populations displayed no significant difference.

The QTL detection using both Interval Mapping and one-way ANOVA demonstrated that the 3 transferred segments displayed an effect on the resistance components (Table 2). The segment on P5 displayed the most important effect on the 3 resistance components. The P2 and the P10 segments displayed both a moderate effect, on REC for the former, on REC by Interval Mapping and on IND by ANOVA for the latter. The favourable allele at P5 originated from Perennial for REC and STA, but from YW for IND. The 
Table 2. QTL detected in the $\mathrm{BC}_{1} \mathrm{~S}_{1} \mathrm{AE}$ validation population

\begin{tabular}{|c|c|c|c|c|c|c|c|c|c|c|c|}
\hline \multirow[t]{2}{*}{ Chr } & \multirow[t]{2}{*}{ Trait $^{9}$} & \multicolumn{3}{|l|}{ ANOVA } & \multicolumn{7}{|c|}{ Interval Mapping } \\
\hline & & Marker ${ }^{1}$ & $\mathrm{P}^{2}$ & f.a. ${ }^{3}$ & $\operatorname{Psn}^{4}$ & $\mathrm{LOD}^{5}$ & $a^{6}$ & $d^{6}$ & $\mathrm{R}^{2} \%^{7}$ & $|d / a|^{8}$ & f.a. ${ }^{3}$ \\
\hline $\mathrm{P} 2$ & REC & - & - & - & 52.99 & 2.63 & 0.78 & -2.10 & 0.21 & 2.69 & $\mathrm{P}$ \\
\hline \multirow[t]{6}{*}{ P5 } & REC & ASC037 & $<0.0001$ & $\mathrm{P}$ & 25.99 & 71.12 & 2.08 & -0.84 & 22.39 & 0.40 & $\mathrm{P}$ \\
\hline & & E43M53-159y & $<0.0001$ & $\mathrm{P}$ & & & & & & & \\
\hline & IND & ASC037 & $<0.0001$ & $\mathrm{Y}$ & 23.99 & 24.52 & -0.19 & 0.09 & 84.68 & 0.50 & $\mathrm{Y}$ \\
\hline & & E43M53-159y & $<0.0001$ & $\mathrm{Y}$ & & & & & & & \\
\hline & STA & ASC037 & $<0.0001$ & $\mathrm{P}$ & 29.99 & 48.52 & 0.91 & -0.78 & 71.05 & 0.86 & $\mathrm{P}$ \\
\hline & & E43M53-159y & $<0.0001$ & $\mathrm{P}$ & & & & & & & \\
\hline \multirow[t]{2}{*}{ P10 } & REC & - & - & - & 38.26 & 2.27 & -0.55 & -2.70 & 0.55 & 4.91 & $\mathrm{Y}$ \\
\hline & IND & E35M61-114y & 0.0173 & $\mathrm{P}$ & & & & & & & \\
\hline
\end{tabular}

${ }^{1}$ marker found significant using 1-way ANOVA; ${ }^{2} \mathrm{P}$ : significance of the marker effect; ${ }^{3}$ f.a.: favourable allele, P: Perennial, Y: Yolo Wonder; ${ }^{4}$ Psn: most likely position of the QTL along the chromosome; ${ }^{5} \mathrm{LOD}$ : LOD value; ${ }^{6} \mathrm{a}$, d: additive, dominance effect; ${ }^{7} \mathrm{R}^{2}$ : percentage of variation explained by markers for the QTL; ${ }^{8} \mathrm{Id} / \mathrm{al}$ : dominance ratio $\left(\mid \mathrm{d} / \mathrm{al}<1\right.$ : partially dominant, $\mid \mathrm{d} / \mathrm{al}>1$ : super-dominant); ${ }^{9} \mathrm{REC}$, receptivity $\left(\mathrm{mm} \cdot \mathrm{d}^{-1}\right)$, IND, inducibility $\left(\mathrm{mm} \cdot \mathrm{d}^{-2}\right)$, STA, stability $\left(\mathrm{mm} \cdot \mathrm{d}^{-1}\right)$.

Table 3. Calculated phenotypic means of the 3 resistance components for the 8 genotypic classes in the $\mathrm{BC}_{1} \mathrm{~S}_{1_{-}} \mathrm{AE}$ validation population

\begin{tabular}{|c|c|c|c|c|c|c|c|c|}
\hline ASC031 (P2) & E43M53-159y (P5) & E35M61-114y (P10) & REC & & IND & & STA & \\
\hline $\mathrm{Y}$ & $\mathrm{Y}$ & $\mathrm{Y}$ & 6.023 & A & -0.744 & $\mathrm{BC}$ & 0.883 & $\mathrm{~A}$ \\
\hline $\mathrm{Y}$ & $\mathrm{Y}$ & $\mathrm{P}$ & $5.583 * *$ & $\mathrm{AB}$ & -0.723 & $\mathrm{ABC}$ & 0.477 & $\mathrm{AB}$ \\
\hline $\mathrm{P}$ & $\mathrm{Y}$ & $\mathrm{Y}$ & $5.467 *$ & $\mathrm{AB}$ & -0.661 & $\mathrm{ABC}$ & 0.860 & $\mathrm{AB}$ \\
\hline $\mathrm{P}$ & $\mathrm{Y}$ & $\mathrm{P}$ & 6.010 & A & -0.756 & $\mathrm{C}$ & 0.852 & $\mathrm{AB}$ \\
\hline $\mathrm{Y}$ & $\mathrm{P}$ & $\mathrm{Y}$ & $4.704 *$ & $\mathrm{ABC}$ & $-0.577 *$ & $\mathrm{AB}$ & 0.587 & $\mathrm{AB}$ \\
\hline $\mathrm{Y}$ & $\mathrm{P}$ & $\mathrm{P}$ & $4.083 *$ & $\mathrm{C}$ & -0.592 & $\mathrm{ABC}$ & 0.076 & $\mathrm{~B}$ \\
\hline $\mathrm{P}$ & $\mathrm{P}$ & $\mathrm{Y}$ & $4.265 * * *$ & $\mathrm{BC}$ & $-0.556 *$ & A & $0.395 *$ & $\mathrm{AB}$ \\
\hline $\mathrm{P}$ & $\mathrm{P}$ & $\mathrm{P}$ & $4.583 * * *$ & $\mathrm{BC}$ & $-0.631 *$ & $\mathrm{ABC}$ & $0.220 *$ & $\mathrm{AB}$ \\
\hline
\end{tabular}

The stars indicate a significantly different mean from the phenotypic mean of the plant carrying the 3 YW alleles at the QTLs on P2, P5, P10 with a type-I-error of $5 \%$ : $* 0.01<\mathrm{P}<0.05$, $* * 0.0001<\mathrm{P}<0.01$, *** $\mathrm{P}<0.0001$. The means indicated with the same letter were not different for a type I error of $5 \%$ using the multiple mean comparison performed with Duncan test.

favourable allele at P10 originated from Perennial for IND, but from YW for REC. The transferred alleles at the QTLs on P2 and P10 displayed a dominant expression whereas the transferred alleles at the major effect QTL on P5 mostly displayed an additive expression for the 3 resistance components.

Plants were arranged in 8 genotypic classes according to their genotypes at P2, P5, and P10-QTL markers. The phenotypic means of the 8 genotypic classes were calculated (Table 3 ). The plants possessing the Perennial alleles at the QTLs on P5 showed for REC and STA a significantly more resistant phenotype than the plants carrying YW alleles on P5, but were not significantly different whatever the allelic combination at the 2 other QTLs.

\section{Validation of the epistatic relationship}

Epistatic effects due to the digenic interaction between the QTLs on P5 and on P10 were reported in the initial mapping F2 population (Thabuis et al. 2003). They were validated in the $\mathrm{BC}_{1} \mathrm{~S}_{1}$ EE population where the digenic interaction between ASC037 (P5) and ASC035p (P10) was found significant for REC $\left(\mathrm{P}=0.0195, \mathrm{R}^{2}=9.44 \%\right)$ and STA $(\mathrm{P}=0.0347$, $\left.\mathrm{R}^{2}=9.40 \%\right)$. The comparison of the phenotypic means of the 9 genotypic classes at both segments clearly displayed an additive $\times$ additive epistatic effect for REC and STA (Figure 4). The Perennial homozygous genotypes at both QTLs significantly displayed the most resistant values.

\section{Recovering the recipient parent genome}

Initiating the MAB from a larger population size than the minimum theoretical size enabled us to apply a significant selection pressure for the background selection step. The number of plants possessing the resistant allele at the markers of the 3 target genome segments varied from 5 to 8 depending on the selec- 

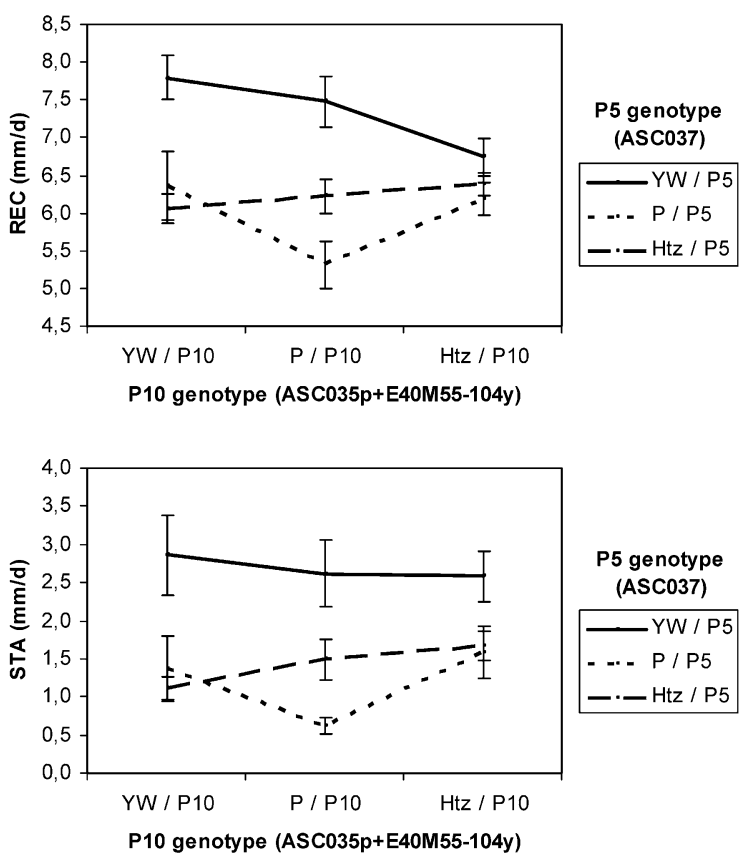

Figure 4. Phenotypic means of the different genotypic classes for $\mathrm{REC}$ and STA in the $\mathrm{BC}_{1} \mathrm{~S}_{1}$ EE validation population. Note: ' $\mathrm{YW}$ ' represents the homozygous Yolo Wonder locus, 'P' the homozygous Perennial locus and 'Htz' the heterozygous locus. REC and STA are expressed in millimeter per day.

tion cycle, enabling a background selection rate of $1 / 5$ to $1 / 8$ (Table 1). The use of precision graphical genotypes enabled to estimate accurately the recipient genome contents (RGC) of the plants. Return to the recipient parent on the non-carrier chromosomes was very efficient as RGC increased from $43.76 \%$ in the parental donor line DH285 to $97.66 \%$ for the selected $\mathrm{BC}_{3}$ plant (Table 4, Figure 2). The return to $\mathrm{YW}$ genome on the carrier chromosomes was significant but lower. It was limited to the extremity of the shorter chromosomes P5 and P10, and more important on the longest chromosome $\mathrm{P} 2$ of which $2 / 3$ was estimated to be homozygous for the recipient parent alleles.

\section{Genetic advance for the horticultural traits}

Analysis of the whole data set showed a strong location effect (15\% to $55 \%$ of the total variation according to the trait) and a year effect (5\% of the total variation). Significant interactions between backcross populations and locations and between backcross populations and years also occurred. Consequently, the 4 trials were analysed independently. For each trial and for all the horticultural traits, the MAB cycle effect was always found highly significant $(13 \%$ to $50 \%$ of the total variation depending on the trait) whereas the block effect and the interaction between MAB cycle and block was rarely significant or explained less than $1 \%$ of the total variation.

The genetic advances for 7 horticultural traits evaluated were estimated for each trial (Table 5). They were all significant and in good agreement across the locations and the years. The strongest improvement was observed for AFW (average fruit weight) and FS (fruit shape). Comparing the horticultural advance between the 3 MAB cycles, the improvement toward the large fruited genotype was continuous. The AFW and FT (fruit flesh thickness) increased significantly, reaching respectively 70 to $73 \%$ and 86 to $100 \%$ of the recipient parent. The FS and IL (internode length) decreased more progressively but also reached the values of the recipient parent. As expected, the genetic advance observed in $\mathrm{BC}_{2}$ was generally larger than that observed in $\mathrm{BC}_{3}$.

\section{Discussion}

Optimisation of the MAB strategy

Taking into account the confidence intervals of the QTL positions by using several markers contributes to the optimisation for a successful QTL transfer. Hospital and Charcosset (1997) derived optimal marker positions, but noted that small shifts from this optimal marker spacing in a MAB program hardly decrease the probability of transferring the QTL. They suggested that the number of markers should be large enough with respect to the length of the confidence interval. In the present study, because of a limited choice of markers, markers roughly located in the confidence interval were selected and still proved successful for transferring the 3 segments. Robert et al. (2001) also displayed a successful MAB program by taking into account the QTL confidence interval. A particular aspect of the present study was the transfer of 2 linked QTLs within a single segment. No optimisation regarding the optimal marker spacing was available in such a case. As these QTLs both displayed a major effect on the resistance (Thabuis et al. 2003), we considered one single interval controlled by 3 markers to transfer the whole segment.

The population size of 350 individuals used here enabled to perform both efficient control of the QTLs, and efficient background selection, as in the study of 
Table 4. Percentage of the Yolo Wonder parent based on molecular data.

\begin{tabular}{lll}
\hline & QTL-carrier chromosomes & Non QTL-carrier chromosomes \\
\hline $\mathrm{DH} 285$ & $3.69 \%$ & $43.76 \%$ \\
The plant $\mathrm{BC}_{1}(3)$ & $14.53 \%$ & $67.98 \%$ \\
The plant $\mathrm{BC}_{2}(3)$ & $21.58 \%$ & $90.45 \%$ \\
The plant $\mathrm{BC}_{3}(3)$ & $25.55 \%$ & $97.66 \%$ \\
\hline
\end{tabular}

Table 5. Efficiency of the return to the recipient parent based on horticultural traits evaluated in 2002

\begin{tabular}{|c|c|c|c|c|c|c|c|c|c|c|c|c|}
\hline & \multicolumn{3}{|c|}{$\mathrm{FS}^{1}$} & \multicolumn{3}{|c|}{$\mathrm{FT}^{2}$} & \multicolumn{3}{|c|}{$\mathrm{AFW}^{3}$} & \multicolumn{3}{|c|}{$\mathrm{IL}^{4}$} \\
\hline & $M^{5}$ & $\%^{6}$ & $\Delta \mathrm{G}^{7}$ & M & $\%$ & $\Delta \mathrm{G}$ & M & $\%$ & $\Delta \mathrm{G}$ & M & $\%$ & $\Delta \mathrm{G}$ \\
\hline \multicolumn{13}{|l|}{ SP02 } \\
\hline $\mathrm{BC}_{1}$ & $2.13 \mathrm{a}^{* * * *}$ & 64 & & $5.51 \mathrm{a}^{* * *}$ & 81 & & $66.18 \mathrm{a}^{* * * *}$ & 40 & & $2.99 \mathrm{a}^{\mathrm{ns}}$ & 0 & \\
\hline $\mathrm{BC}_{2}$ & $1.53 \mathrm{~b}^{* * *}$ & 86 & -28 & $6.62 b^{n s}$ & 97 & +17 & $86.95 \mathrm{~b}^{* * * *}$ & 53 & +24 & $2.87 \mathrm{a}^{\mathrm{ns}}$ & 39 & -4 \\
\hline $\mathrm{BC}_{3}$ & $1.35 \mathrm{c}^{\mathrm{ns}}$ & 93 & -12 & $7.20 \mathrm{c}^{\mathrm{ns}}$ & 107 & +8 & $120.43 \mathrm{c}^{* * * *}$ & 73 & +28 & $2.56 \mathrm{a}^{\mathrm{ns}}$ & 138 & -11 \\
\hline DH285 & 3.86 & & & 1.75 & & & 2.92 & & & 2.99 & & \\
\hline YW & 1.17 & & & 6.85 & & & 164.64 & & & 2.68 & & \\
\hline \multicolumn{13}{|l|}{ IT02 } \\
\hline $\mathrm{BC}_{1}$ & $1.69 \mathrm{a}^{* * * k}$ & 71 & & $4.05 \mathrm{a}^{* * * *}$ & 72 & & $68.68 \mathrm{a}^{* * * *}$ & 43 & & $1.65 \mathrm{a}^{\mathrm{ns}}$ & -67 & \\
\hline $\mathrm{BC}_{2}$ & $1.32 \mathrm{~b}^{\mathrm{ns}}$ & 88 & -22 & $4.53 \mathrm{~b}^{*}$ & 81 & +10 & $82.49 \mathrm{~b}^{* * * k}$ & 51 & +17 & $1.38 \mathrm{a}^{\mathrm{ns}}$ & -8 & -16 \\
\hline $\mathrm{BC}_{3}$ & $1.08 \mathrm{c}^{\mathrm{ns}}$ & 99 & -18 & $4.82 \mathrm{~b}^{*}$ & 86 & +6 & $112.97 \mathrm{c}^{* * * *}$ & 70 & +27 & $1.32 \mathrm{a}^{\mathrm{ns}}$ & 25 & -5 \\
\hline DH285 & 3.22 & & & 1.55 & & & 3.67 & & & 1.41 & & \\
\hline YW & 1.06 & & & 5.62 & & & 161.4 & & & 1.05 & & \\
\hline
\end{tabular}

${ }^{1}$ Fruit Shape, ${ }^{2}$ Flesh Thickness, ${ }^{3}$ Average Fruit Weight, ${ }^{4}$ Internode Length, ${ }^{5}$ Mean of the horticultural trait, ${ }^{6}$ percentage of return to the recipient parent calculated as $100 *\left(\mathrm{BC}_{\mathrm{n}}-\mathrm{DH} 285\right) /(\mathrm{YW}-\mathrm{DH} 285)$, where $\mathrm{BCn}$, DH285 and $\mathrm{YW}$ are the phenotypic values of the corresponding population and genotypes. ${ }^{7}$ Genetic advance per cycle calculated as $100 *\left(\mathrm{BC}_{\mathrm{n}+1}-\mathrm{BC}_{\mathrm{n}}\right) / \mathrm{BC}_{\mathrm{n}}$; SP02 is the trial performed in Spain in 2002 and IT02 in Italy in 2002. The means indicated with the same letter were not different to the ones from the others for a type I error of 5\% using the multiple mean comparison performed with Duncan test. The stars indicate a mean significantly different from the mean of YW: $*$ $0.01<\mathrm{P}<0.05$, ** $0.0001<\mathrm{P}<0.01$, *** $\mathrm{P}<0.0001$, and ns means non significantly different from ' $\mathrm{YW}$ ' mean (P $>0.05$ ).

Bouchez et al. (2002) where several plants could be selected carrying the donor alleles along the transferred intervals. By contrast, Lawson et al. (1997), which aimed at transferring in cultivated tomato alleles at 5 QTLs originating from a wild accession were unsuccessful in screening a single plant with the 5 favourable alleles because they used too small population size (150 to 273 plants).

Increasing the population size drastically increased the genotyping effort. However, the sequential genotyping approach used decreased strongly the experimental expense and proved to be successful for QTL transfer as well as for major genes (Frisch and Melchinger 2001).

\section{Additive and epistatic effects of the transferred segments}

We compared the QTL detection performed in the validation populations and in the $\mathrm{DH}$ mapping population (Thabuis et al. 2003). The larger size of the validation population and the change of genetic background towards YW increased the accuracy of the QTL effect and location estimation, and consequently revealed the true breeding value of the QTLs. The changes in QTL effect and position according to the population size were well illustrated by Melchinger et al. (1998) that experimentally showed that small population size for QTL detection was responsible for false positive QTL mapping, an overestimation of QTL effect and to biases in QTL positions. Considering the 2 linked QTLs located on P5 in the DH mapping population, only a single QTL was re-detected in the $\mathrm{BC}_{1} \mathrm{~S}_{1} \mathrm{AE}$ population. This resistance factor still displayed a strong effect on all the resistance components. It remained located in the same genomic region as in the DH mapping population. The minor effect QTLs on P2 and P10 were also re-detected in the $\mathrm{BC}_{1} \mathrm{~S}_{1}$ AE population, although their $\mathrm{R}^{2}$ values were lower. A distinct position was detected for the QTL mapped on P2. Such differences observed for QTL effects and positions could be related to their 
weak phenotypic effects. Discrepancies were observed in the sign of the QTL effects after introgression, when compared to those expected from the initial DH mapping population, as observed by Bouchez et al. (2002) that inferred the genotype-byenvironment interaction effects. Discrepancies could also be due to the QTL-by-genetic background interaction. The magnitude of the epistatic interaction was also lower in the $\mathrm{BC}_{1} \mathrm{~S}_{1-}$ EE population compared to the DH mapping population, and the epistatic region on P10 was found at a slightly different location.

The shifts in QTL detection between mapping populations and validation breeding pointed out the necessity of optimisations for the QTL transfer by taking into account the confidence interval. The relatively small population size for the QTL mapping step represented the major pitfall and led to a 'cautious optimism' regarding marker-assisted selection (Young 1999; Dekkers and Hospital 2002). This cause might explain the inconsistent results reported for several QTL validation studies (Han et al. 1997; Toojinda et al. 1998; Romagosa et al. 1999; Zhu et al. 1999). Other causes such as QTL $\times$ environment interaction effects (Bouchez et al. 2002) and the change in the genetic background (Lawson et al. 1997) might also decrease MAS efficiency.

\section{Efficiency of the return to the recipient parent}

The necessity to retain the plants carrying all the transferred segments slowed down the return to the recipient parent genome. After only $3 \mathrm{MAB}$ cycles, all the non-carrier chromosomes were converted to the recipient genome. Hospital and Charcosset (1997) showed that the background selection is efficient until the third backcross generation and that the gain would be limited to the carrier chromosomes in the next MAB cycles. However in our study, the few plants retained from the foreground selection step did not permit to accelerate the return to $\mathrm{YW}$ on the QTL-carrier chromosomes. To achieve this objective, a population size up to 600 or 800 individuals would be necessary according to Hospital and Charcosset's simulations (1997).

A significant return to the recipient parent was observed along the $3 \mathrm{MAB}$ cycles. In the $\mathrm{BC}_{3}$ population, the horticultural fruit traits recovered on average $70 \%$ to $100 \%$ of YW values displaying the phenotypic impact of the marker-assisted selection for the genetic background. Similar results were obtained with molecular data with $25.55 \%$ of YW alleles over the QTL-carrier chromosomes and $97.66 \%$ over the non-carrier chromosomes, averaging $79.63 \%$ of YW alleles over the whole genome $([3 * 25.55+9 * 97.66 \%] / 12)$.

For AFW, a significant difference with YW still occurred in $\mathrm{BC}_{3}$ with an average value of $70.34 \%$ of the recipient parent. This slow genetic advance could in part be explained by the co-location observed between the transferred resistance QTL on P10 and a QTL involved in fruit weight (Ben Chaim et al. 2001). This antagonism was highly likely to occur in our cross since the small-fruited parent is the same in both studies. However, this fruit weight QTL was shown to display a moderate effect that should weakly affect the horticultural advance. This suggests that the effect of this fruit weight QTL was higher in the recipient parent YW or that other unfavourable linkages occurred on P2 and P5. From this program, NILs (near-isogenic lines) for each of the resistance QTLs were produced. Additional markers will be located in those genomic regions in order to develop fine mapping experiments of the major P5-QTL and to analyse linkage with horticultural traits.

\section{Acknowledgements}

This work and A. Thabuis were financially supported by 'Vilmorin, Clause and Cie' and 'Seminis Vegetable Seed'. The authors thank deeply the breeders C. Basterreix, J.W. Hennart and C. Robledo for their help in the program management. The authors also thank A. Blattes, V. de Conto, M. Havard and M. Moreau for their valuable help in the genotyping work, and T. Phaly, G. Nemouchy and D. Potey for the progeny production and experimental trials.

\section{References}

Basten C.J., Weir B.S., Zeng Z.B. 1997. QTL cartographer: a reference manual and tutorial for QTL mapping. Department of statistics, North Carolina state University, Raleigh, North Carolina, USA.

Ben Chaim A., Paran I., Grube R.C., Jahn M., van Wijk R., Peleman J. 2001. QTL mapping of fruit-related traits in pepper. Theor. Appl. Genet. 102: 1016-1028.

Bouchez A., Hospital F., Causse M., Gallais A., Charcosset A. 2002. Marker-assisted introgression of favorable alleles at quantitative trait loci between maize elite lines. Genetics 162: 19451959.

Clerjeau M., Pitrat M., Nourisseau J.G. 1976. La résistance du piment (Capsicum annuum) à Phytophthora capsici. IV. Etude de 
l'agressivité de divers isolats au niveau des feuilles, des tiges et du collet des plantes résistantes et sensibles. Ann Phytopathol. 8: 411-423.

Dekkers J.C.M., Hospital F. 2002. The use of molecular genetics in the improvement of agricultural populations. Nature Reviews Genetics 3: 22-32.

Frisch M., Melchinger A.E. 2001. Marker-assisted backcrossing for introgression of a recessive gene. Crop. Sci. 41: 1485-1494.

Fulton T.M., Chunwongse J., Tanksley S.D. 1995. Microprep protocol for extraction of DNA of tomato and other herbaceous plants. Plant Mol. Biol. Report. 13(3): 207-209.

Han F., Romagosa I., Ullrich S.E., Jones B.L., Hayes P.M., Wesenberg D.M., 1997. Molecular marker-assisted selection for malting quality traits in barley. Mol. Breed. 3: 427-437.

Hospital F., Charcosset A. 1997. Marker-assisted introgression of quantitative trait loci. Genetics 147: 1469-1485.

Lander E., Green P., Abrahamson J., Barlow A., Daley M., Lincoln S., Newburg L. 1987. Mapmaker: an interactive package for constructing primary genetic linkage maps of experimental and natural populations. Genomics 1: 174-181.

Lawson D.M., Lunde C.F., Mutschler M.A. 1997. Marker-assisted transfer of acylsugar-mediated pest resistance from the wild tomato, Lycopersicon pennellii, to the cultivated tomato, Lycopersicon esculentum. Mol. Breed. 3: 307-317.

Lefebvre V, Palloix A. 1996. Both epistatic and additive effects of QTLs are involved in polygenic induced resistance to disease: a case study, the interaction pepper-Phytophthora capsici Leonian. Theor. Appl. Genet. 93: 503-511.

Lefebvre V., Palloix A., Rives M. 1993. Nuclear RFLP between pepper cultivars (Capsicum annuит L.). Euphytica 71: 189-199.

Lefebvre V., Palloix A., Caranta C., Pochard E. 1995. Construction of an intraspecific integrated linkage map of pepper using molecular markers and doubled-haploid progenies. Genome 38: $112-121$.

Lefebvre V., Goffinet B., Chauvet J- C, Caromel B., Signoret P., Brand R., Palloix A. 2001. Evaluation of genetic distances between pepper inbred lines for cultivar protection purposes: comparison of AFLP, RFLP and phenotypic data. Theor. Appl. Genet. 102: 741-750.

Lefebvre V., Pflieger S., Thabuis A., Caranta C., Blattes A., Chauvet J.C., Daubèze A.M., Palloix A. 2002. Towards the saturation of the pepper linkage map by alignment of three intraspecific maps including known-function genes. Genome 45: 839-854.

Melchinger A.E., Utz H.F., Schon C.C. 1998. Quantitative trait locus (QTL) mapping using different testers and independent population samples in maize reveals low power of QTL detection and large bias in estimates of QTL effects. Genetics 149: 383-403.

Palloix A., Daubèze A.M., Pochard E. 1988. Time sequences of root infection and resistance expression in an artificial inocula- tion method of pepper with Phytophthora capsici. J. Phytopathol. 123: 12-24.

Palloix A., Daubèze A.M., Phaly T., Pochard E. 1990. Breeding transgressive lines of pepper for resistance to Phytophthora capsici in a recurrent selection system. Euphytica 51: 141-150.

Palloix A., Daubèze A.M., Lefebvre V., Caranta C., Moury B., Pflieger S., Gebre-Selassie K., Marchoux G. 1997. Constructing resistant genotypes fitting cultivation conditions in pepper. C. R. Acad. Agric. Fr. 7: 87-98.

Pochard E., Daubèze A.M. 1980. Recherche et évaluation des composantes d'une résistance polygénique : la résistance du piment à Phytophthora capsici. Ann. Amélior. Plant. 26: 377-398.

Pochard E., Clerjeau M., Pitrat M. 1976. La résistance du piment, Capsicum annuum L., à Phytophthora capsici Leon. I. Mise en évidence d'une induction progressive de la résistance. Ann. Amélior. Plant. 26: 35-50.

Robert V.J.M., West M.A.L., Inai S., Caines A., Arntzen L., Smith J.K., St. Clair D.A. 2001. Marker-assisted introgression of blackmold resistance QTL alleles from wild Lycopersicon cheesmanii to cultivated tomato (L. esculentum) and evaluation of QTL phenotypic effects. Mol. Breed. 8: 217-233.

Romagosa I., Han F., Ullrich S.E., Hayes P.M., Wesenberg D.M., 1999. Verification of yield QTL through realized molecular marker-assisted selection responses in a barley cross. Mol Breed. 5: 143-152.

SAS Institute Inc., 1989. SAS/STAT user's guide, version $6,4^{\text {th }}$ edition. SAS Institute Inc, Cary, North Carolina, USA.

Servin B., Dillman C., Decoux G., Hospital F. 2002. MDM: a program to compute fully informative genotype frequencies in complex breeding schemes. J. Hered. 93(3): 227-228.

Thabuis A., Palloix A., Pflieger S., Daubèze A.M., Caranta C., Lefebvre V., 2003. Comparative mapping of Phytophthora resistance loci in pepper germplasm: evidence for conserved resistance loci across Solanaceae and for a large genetic diversity. Theor. Appl. Genet. 106: 1473-1485.

Toojinda T., Baird E., Booth A., Broers L., Hayes P., Powell W., Thomas W., Vivar H., Young G., 1998. Introgression of quantitative trait loci (QTLs) determining stripe rust resistance in barley: an example of marker-assisted line development. Theor. Appl. Genet. 96: 123-131.

Vos P., Hogers R., Bleeker M., Reijans M,. van de Lee T., Hornes M., Frijters A., Pot J., Peleman J., Kuiper M., Zabeau M. 1995. AFLP: a new technique for DNA fingerprinting. Nucleic. Acid. Research 23(21): 4407-4417.

Young N.D., 1999. A cautiously optimistic vision for marker-assisted breeding. Mol. Breed. 5: 505-510.

Zhu H., Briceno G., Dovel R., Hayes P.M., Liu B.H., Liu C.T., Ullrich S.E., 1999. Molecular breeding for grain yield in barley: an evaluation of QTL effects in a spring barley cross. Theor. Appl. Genet. 98: 772-779. 